\title{
Stability of the Renormalization Group in the 2D Random Ising and Baxter Models with respect to the Replica Symmetry Breaking
}

\author{
D.E.Feldman, A.V.Izyumov and Viktor Dotsenko \\ Landau Institute for Theoretical Physics, \\ Russian Academy of Sciences, \\ Kosygina 2, 117940 Moscow, Russia
}

\begin{abstract}
We study the critical properties of the weakly disordered two-dimensional Ising and Baxter models in terms of the renormalization group (RG) theory generalized to take into account the replica symmetry breaking (RSB) effects. Recently it has been shown that the traditional replica-symmetric RG flows in the dimensions $D=4-\epsilon$ are unstable with respect to the RSB potentials and a new spin-glass type critical phenomena has been discovered [11, [12]. In contrast, here it is demonstrated that in the considered two-dimensional systems the renormalization-group flows are stable with respect to the RSB modes. It is shown that the solution of the renormalization group equations with arbitrary starting RSB coupling matrix exhibits asymptotic approach to the traditional replica-symmetric ones. Thus, in the leading order the non-perturbative RSB degrees of freedom does not effect the critical phenomena in the two-dimensional weakly disordered Ising and Baxter models studied earlier.
\end{abstract}


The effects produced by weak quenched disorder on the critical phenomena near the phase transition point have been studied since many years ago [1]-[6]. According to the Harris criterion [1], the disorder effects the critical behaviour only if $\alpha$, the specific heat exponent of the pure system, is positive. In this case a new universal critical behavior, with new critical exponents, is established sufficiently close to the phase transition point for $\left(T / T_{c}-1\right) \equiv \tau \ll \tau_{u} \equiv u^{1 / \alpha}[2]$, where $u \ll 1$ is the parameter which describes the strength of the disorder. In contrast, when $\alpha<0$, the disorder appears to be irrelevant for the critical behavior.

Originally the modified critical behaviour has been derived for the classical $\phi^{4}$ model near four dimensions [2], and later it has been studied for the two-dimensional Ising [3], Baxter [4] and Potts [5] models by various renormalization group (RG) techniques, and by numerical simulations [6].

In dealing with the quenched disorder the traditional approach is the replica method, and in terms of replicas all the results obtained for the systems listed above correspond to the so-called replica-symmetric (RS) solutions. Physically it means that olny unique ground state is assumed to be relevant for the observable thermodynamics. The problem, however, is that in the presence of the quenched disorder there exist numerous local minimum configurations separated by finite barriers, and in this case the direct application of the traditional replica-symmetric RG scheme may be questioned.

On the other hand, it is the Parisi Replica Symmetry Breaking (RSB) scheme which has been developed specifically for dealing with disordered systems which exhibit numerous local minima states (see e.g. [7]). Recent studies show that besides mean-field theory of spin-glasses the RSB approach can also be generalized for situations where one has to deal with fluctuations as well [8], [9], [10]. In the paper [11 qualitative arguments were presented demonstrating the mechanism how the summation over multiple local minima configurations could provide additional non-trivial RSB interaction potentials for the fluctuating fields.

One can hope that such type of the generalized RG scheme self-consistently takes into account relevant degrees of freedom coming from the numerous local minima, and in particular, the instability of the RS fixed point with respect to the RSB would indicate that the multiplicity of the local minima is relevant for the critical properties in the fluctuation region. And inversely, if the traditional RS RG flows turn out to be stable with respect to the RSB modes, then it can be concluded that such non-perturbative degrees of freedom are irrelevant for the critical behaviour in a considered system.

The first example of replica symmetry broken solutions in the renormalization group has been suggested in [13] in the context of the 2D random field XY-model, where instability of the RG flows with respect to the RSB modes has been discovered. Similar phenomena has been observed in the weakly disordered classical ferromagnet (the $\phi^{4}$ theory) in the dimension $D=4-\epsilon$ [11]. Here the RSB degrees of freedom produce dramatic effect on the asymptotic behaviour of the RG flows, such that for a general type of the RSB there exist no stable fixed points, and the RG arrives into the strong coupling regime at the finite scale $(\sim \exp (-1 / u))$ [12.

On the other hand, similar considerations for weakly disordered 2D Potts model shows that, although the traditional RS fixed point also turns out to be unstable, there 
exist stable non-trivial fixed point characterized by the continuous RSB structure of the coupling matrix [14].

In this Letter we report on the RSB solution for the two-dimensional Ising and Baxter models with random bonds. We consider the models with spin couplings having small fluctuations around a mean ferromagnetic value. This gives a possibility to study the model in the continuum limit, because one approaches the critical point sufficiently close before the randomness becomes relevant. For the two-dimensional Ising and Baxter models this allows to use the renormalization group based on the fermion representation.

In contrast to the previous studies, in the considered systems the RG flows turns out to be stable with respect to RSB modes. The explicit solution of the corresponding RG equations shows that for any starting RSB structure of the coupling matrix the asymptotic solutions become replica-symmetric. It means that the RSB ("non-perturbative") degrees of freedom appear to be irrelevant for the critical phenomena in these systems, and they exhibit the usual replica symmetric critical behavior which has been studied earlier [3], [4].

\section{The Ising Model}

It is well known that the two-dimensional Ising model in the critical region is equivalent to the problem of the free fermions [15]. The effect of the quenched disorder can be described by the random contribution to the effective temperature $\tau$. Thus, the random bond Ising model in the critical region can be reduced to the field theory with the following Lagrangian:

$$
L[\psi]=-\frac{1}{2} \int d^{2} x[\bar{\psi} \hat{\partial} \psi+(\tau+\delta \tau) \bar{\psi} \psi],
$$

where $\delta \tau$ is the Gaussian random variable with $\overline{\left(\delta \tau^{2}\right)}=2 u \ll 1$, and $\psi$ and $\bar{\psi}=i \psi \hat{\sigma}_{y}$ are the two-component real fermion fields.

The averaging over quenched random variable $\delta \tau$ can be performed in terms of the standard replica procedure. The resulting replica Lagrangian has the following form:

$$
L_{n}[\psi]=-\int d^{2} x\left[\frac{1}{2} \sum_{a=1}^{n} \bar{\psi}^{a}(\hat{\partial}+\tau) \psi^{a}-\frac{1}{4} \sum_{a, b=1}^{n} g_{a b} \bar{\psi}^{a} \psi^{a} \bar{\psi}^{b} \psi^{b}\right],
$$

In the usual replica-symmetric theory the coupling matrix does not depend on the replica indices: $g_{a b}=u$. Then, in terms of the standard RG scheme one can easily calculate the critical properties of the system, and in particular for leading singularity of the specific heat one finds: $C(\tau) \sim \log \log (1 /|\tau|)$ [3].

According to the discussion in the introductory part of this Letter, in our present scenario we assume that due to non-perturbative effects the replica symmetry in the coupling matrix $g_{a b}$ can be broken. In other words, the starting point of the further analysis is the assumption that the matrix $g_{a b}$ has the Parisi-type structure (see e.g. [[]]).

The corresponding (one-loop) RG equations for the replica matrix $g_{a b}$ and for the mass parameter $\tau$ are: 


$$
\begin{aligned}
& \frac{d g_{a b}}{d \xi}=\frac{1}{\pi} \sum_{c \neq a, b} g_{a c} g_{c b} . \\
& \frac{d \log \tau}{d \xi}=\frac{1}{\pi} \sum_{b \neq 1} g_{1 b} .
\end{aligned}
$$

where $\xi$ is the standard $R G$ rescaling parameter.

In terms of the Parisi RSB scheme [7] in the limit $n \rightarrow 0$ the matrix $g_{a \neq b}$ is parametrized by the function $g(x)$ defined in the interval $0 \leq x \leq 1$ (replica-symmetric situation corresponds to $g(x)=$ const, independent of $x$ ). According to the standard technique of the Parisi RSB algebra [9] (see also [11, [12]) the equation (3) can be represented as follows:

$$
\frac{d g(x)}{d \xi}=-\frac{1}{\pi}\left[x g^{2}(x)+2 g(x) \int_{x}^{1} d y g(y)+\int_{0}^{x} d y g^{2}(y)\right]
$$

The structure of this equation is similar to that for the $\phi^{4}$-theory with the broken replica symmetry, and it can be solved following the method suggested in [12. Simple calculations yield:

$$
\begin{gathered}
g^{\prime}(x, \xi)=\frac{g^{\prime}(x, 0) \exp \left(-\frac{2}{\pi} \int_{0}^{\xi} d \eta \bar{g}(\eta)\right)}{\left[1+\frac{1}{\pi} \int_{0}^{x} y g^{\prime}(y, 0) d y \int_{0}^{\xi} d \eta \exp \left(-\frac{2}{\pi} \int_{0}^{\eta} d \theta \bar{g}(\theta)\right)\right]^{2}} . \\
g(0, \xi)=g(0,0) \exp \left(-\frac{2}{\pi} \int_{0}^{\xi} \bar{g}(\eta) d \eta\right)
\end{gathered}
$$

where $g^{\prime}(x, \xi) \equiv \frac{d}{d x} g(x, \xi)$ and $\bar{g}(\xi) \equiv \int_{0}^{1} g(x, \xi) d x$.

Equations (6) -(7) allow to study the large scale asymptotic behaviour of the renormalized function $g(x, \xi)$ provided the starting function is given. Simple calculations show that at any value of $x$ in the limit $\xi \rightarrow \infty$ the derivative in (6) tends to zero as $\left[\xi(\log \xi)^{2}\right]^{-1}$, while $\bar{g}(\xi) \sim \xi^{-1}$. It means that the asymptotic renormalized function $g(x, \xi \rightarrow \infty)$ tends to become flat in the whole interval $0<x<1)$. In other words, for any starting RSB function $g(x, \xi=0)$ the asymptotic solution of the RG eq.(5) becomes replica-symmetric.

One can also easily check that slowly decaying corrections to the RS solution does not effect the leading singularity of the specific heat. From the eq.(化) for the renormalized temperature parameter one finds:

$$
\tau(\xi)=\tau(0) \exp \left(-\frac{1}{\pi} \int_{0}^{\xi} \bar{g}(\eta) d \eta\right)
$$

Then, using eqs. (6),(7) and (8) one can derive the following closed form relation on $\tau(\xi)$ :

$$
\left.\log \left(\frac{\tau(0)}{\tau(\xi)}\right)=\frac{g(0,0)}{\pi} \int_{0}^{\xi} d \eta\left(\frac{\tau(\eta)}{\tau(0)}\right)^{2}+O\left(\sqrt{\int_{0}^{\xi} d \eta\left(\frac{\tau(\eta)}{\tau(0)}\right.}\right)^{2}\right) .
$$

In the asymptotic limit $\xi \rightarrow \infty$ one finally gets: 


$$
\tau(\xi) \sim \frac{1}{\sqrt{\xi}}+O\left(\frac{1}{\sqrt{\xi \log \xi}}\right) .
$$

which in the leading order coincides with the RS result. Correspondingly, the leading singularity of the specific heat (which can be estimated as $C(\tau) \sim \int^{\ln (1 /|\tau|)} d \xi \tau^{2}(\xi)$ ) remains the same as in the $\mathrm{RS}$ case [3] as well.

\section{The Baxter Model}

The Baxter model [16] can be formulated in terms of two 2D Ising models coupled by four-spin interactions. The strength of this coupling is described by parameter $\lambda$ (the case of $\lambda=0$ corresponds to two independent 2D Ising models). The pure Baxter model can be solved exactly and in particular, its specific heat exponent is proportional to $\lambda: C_{\text {pure }}(\tau) \sim|\tau|^{-\frac{\lambda}{\pi}}$ (for $|\lambda| \ll 1$ ).

Similar to the weakly disordered 2D Ising system considered above, in the continuum limit near the critical point the corresponding Baxter model can be described in terms of the two-component complex fermion field:

$$
L[\psi]=-\int d^{2} x\left[\frac{1}{2} \bar{\psi} \hat{\partial} \psi+\frac{1}{2}(\tau+\delta \tau(x)) \bar{\psi} \psi-\frac{1}{4} \lambda(\bar{\psi} \psi)(\bar{\psi} \psi)\right]
$$

After averaging over the random function $\delta \tau(x)$ one gets the following replica Lagrangian:

$$
L_{n}[\psi]=-\int d^{2} x\left[\frac{1}{2} \sum_{a=1}^{n} \bar{\psi}^{a}(\hat{\partial}+\tau) \psi^{a}-\frac{1}{4} \sum_{a, b} g_{a b}\left(\bar{\psi}^{a} \psi^{a}\right)\left(\bar{\psi}^{b} \psi^{b}\right)\right]
$$

In the RS case for the replica coupling matrix one has: $g_{a b}=\lambda \delta_{a b}+u$. The critical properties of such system has been studied earlier [4, and it was shown that for $\lambda>0$ (when the specific heat of the pure system is divergent) the leading singularity of the specific heat coincides with that of the random Ising model: $C(\tau) \sim \ln \ln \frac{1}{|\tau|}$. On the other hand, for $\lambda<0$ (when the specific heat of the pure system is finite), the singularity of the specific heat changes to: $C(\tau) \sim|\tau|^{-\frac{\lambda_{*}}{\pi}}$, where $\lambda_{*}=\lambda \exp (-u /|\lambda|)$.

Here we study the situation when the coupling matrix $g_{a b}$ has a general RSB Parisi structure. The corresponding RG equations are:

$$
\begin{gathered}
\frac{d g_{a b}}{d \xi}=-\frac{1}{\pi}\left\{g_{a b}\left(g_{a a}+g_{b b}\right)-2 \sum_{c} g_{a c} g_{c b}\right\} \\
\frac{d(\ln \tau)}{d \xi}=-\frac{1}{\pi}\left\{g_{a a}-2 \sum_{c} g_{a c}\right\}
\end{gathered}
$$

According to the standard technique of the Parisi RSB algebra, in the limit $n \rightarrow 0$ the matrix $g_{a b}$ is parametrized in terms of its diagonal element $\tilde{g}$ and the off-diagonal function $g(x)$. The replica symmetric situation corresponds to the case $g(x)=$ const independent of $x$. After simple algebra, instead of the eqs.(13),(14) in the limit $n \rightarrow 0$ one gets: 


$$
\begin{aligned}
& \frac{d}{d \xi} \tilde{g}=-\frac{2}{\pi} \int_{0}^{1} d x g^{2}(x) \\
& \frac{d}{d \xi} g(x)=\frac{2}{\pi}\left[\tilde{g} g(x)-2 \bar{g} g(x)-\int_{0}^{x} d y[g(y)-g(x)]^{2}\right] \\
& \frac{d}{d \xi}(\ln \tau)=\frac{1}{\pi}(\tilde{g}-2 \bar{g})
\end{aligned}
$$

where $\bar{g} \equiv \int_{0}^{1} d x g(x)$.

The solution of the eqs.(15) can be represented in the same way as in the Ising case considered above (cf eq.(6)):

$$
g^{\prime}(x, \xi)=\frac{g^{\prime}(x, 0) \exp \left\{-\frac{2}{\pi} \int_{0}^{\xi} d \eta(2 \bar{g}(\eta)-\tilde{g}(\eta))\right\}}{\left[1+\frac{2}{\pi} \int_{0}^{x} y g^{\prime}(y, 0) d y \int_{0}^{\xi} d \eta \exp \left\{-\frac{2}{\pi} \int_{0}^{\eta} d \theta(2 \bar{g}(\theta)-\tilde{g}(\theta))\right\}\right]^{2}} .
$$

Using the above equation one can easily study the asymptotic (for $\xi \rightarrow \infty$ ) behaviour of the function $g(x, \xi)$. The behaviour of the solution depends on the sign of the starting parameter $g_{0} \equiv \tilde{g}(\xi=0)-\bar{g}(\xi=0)$ which corresponds to the initial coupling $\lambda$ in the replica symmetric case. Consider the two cases separately.

1) $g_{0}>0$

The corresponding replica-symmetric RG equations have the following asymptotic solution [4]:

$$
\begin{aligned}
& g_{R S} \simeq \frac{2}{\pi}\left[\frac{1}{\xi}-\frac{1}{\xi \ln \xi}\right]+\frac{a}{\xi(\ln \xi)^{2}} \\
& \tilde{g}_{R S} \simeq \frac{2}{\pi}\left[\frac{1}{\xi}-\frac{2}{\xi \ln \xi}\right]+\frac{b}{\xi(\ln \xi)^{2}}
\end{aligned}
$$

where $a$ and $b$ are constants. Then, using eq.(16) for the RSB correction to these solutions: $g(x, \xi)=g_{R S}(\xi)+g_{R S B}(x, \xi)$ and $\tilde{g}(\xi)=\tilde{g}_{R S}(\xi)+\tilde{g}_{R S B}(\xi)$, one immediately finds that

$$
\begin{aligned}
& g_{R S B}^{\prime}(x, \xi) \sim \frac{V(x)}{\xi(\ln \xi)^{2}} \\
& \tilde{g}_{R S B}(\xi) \sim \frac{1}{\xi^{2}(\ln \xi)^{2}}
\end{aligned}
$$

where $V(x)$ is a function which is defined by the starting RSB function $g(x, \xi=0)$. This means that on large scales the RSB deviations are vanishing in comparison with the $\mathrm{RS}$ solution, and the asymptotical properties of the model are not affected by the starting RSB.

2) $g_{0}<0$

In this case the replica-symmetric RG trajectories arrive to the fixed point $\left(g_{*}, \tilde{g}_{*}\right)$, which depends on the initial coupling parameters $g(\xi=0) \equiv g(0)$ and $g_{0}$ [ [4]:

$$
\begin{aligned}
& \tilde{g}_{*}=-\left|g_{0}\right| \exp \left[-\frac{g(0)}{\left|g_{0}\right|}\right] \\
& g_{*}=0
\end{aligned}
$$

Using eqs.(15) or (16) this fixed point can be easily shown to be also stable with respect to general RSB deviations. In other words, the RG trajectories defined by eqs.(15) arrive to the same fixed point $\left(\tilde{g}_{*}, 0\right)$ as in the RS case. 
In conclusion, we have studied the effects of the replica symmetry breaking in the renormalization group for the $2 \mathrm{D}$ random Ising and Baxter models. We have found that the traditional replica-symmetric renormalization group flows are stable with respect to the RSB modes. Thus, unlike the other suggestions [17], we conclude that the effects of possible non-perturbative degrees of freedom in the 2D random Ising and Baxter models must be irrelevant for the critical behaviour studied earlier.

\section{References}

[1] A.B.Harris, J.Phys. C 7, 1671 (1974)

[2] T. C. Lubensky and A. B. Harris, AIP Conf. Proc. 24311 (1974)

G. Grinstein, AIP Conf. Proc. 24313 (1974)

A. B. Harris and T. C. Lubensky, Phys.Rev.Lett., 33, 1540 (1974)

D. E. Khmelnitskii, ZhETF (Soviet Phys. JETP) 68, 1960 (1975)

G. Grinstein and A. Luther, Phys.Rev. B 13, 1329 (1976)

[3] Vik.S.Dotsenko and Vl.S.Dotsenko, JETP Lett., 33, 37 (1981)

Vik.S.Dotsenko and Vl.S.Dotsenko, Adv.Phys. 32, 129 (1983)

B.N.Shalaev, Sov. Phys. Solid State, 26, 1811 (1984)

R.Shankar, Phys.Rev.Lett., 58, 2466 (1986)

A.W.W.Ludwig, Phys.Rev.Lett., 61, 2388 (1988)

[4] Vik.S.Dotsenko and Vl.S.Dotsenko, J. Phys. A: Math.Gen. 17, L301 (1984)

[5] A.W.W.Ludwig, Nucl.Phys. B 285, 97 (1987); Nucl.Phys. B 330, 639 (1990)

Vl.S.Dotsenko, M.Picco and P.Pujol, Phys.Lett. B 347, 113 (1995)

Vl.S.Dotsenko, M.Picco and P.Pujol, Nucl.Phys. B 455, 701 (1995)

[6] V.B.Andreichenko, Vl.S.Dotsenko, W.Zelke and J.-S.Wang, Nucl.Phys. B 344, 531 (1990)

J.-S.Wang, W.Zelke, Vl.S.Dotsenko and V.B.Andreichenko, Europhys.Lett., 11, $301(1990)$

J.-S.Wang, W.Zelke, Vl.S.Dotsenko and V.B.Andreichenko, Physica A., 164, 221 (1990)

W.Zelke, L.N.Shchur and A.L.Talapov, in "Annual Reviews of Computational Physica" (Ed. D.Stauffer), World Scientific, Singapore, 17 (1994)

[7] M.Mezard, G.Parisi, M.Virasoro, "Spin glass theory and beyond", World Scientific, 1987

[8] M.Mezard and G.Parisi, J.Phys. I 1, 809 (1991)

[9] M.Mezard and A.P.Young, Europhys.Lett., 18, 653 (1992) 
[10] S. Korshunov, Phys.Rev. B48, 3969 (1993)

[11] Vik.S.Dotsenko, B.Harris, D.Sherrington and R.Stinchbombe, J.Phys. A: Math.Gen. 28, 3093 (1995)

[12] Vik.S.Dotsenko and D.E.Feldman, J.Phys.A 28, 5183 (1995)

[13] P. Le Doussal and T.Giamarchi, Phys.Rev.Lett., 74, 606 (1995)

[14] Vik.S.Dotsenko, Vl.S.Dotsenko, M.Picco and P.Pujol, Europhys.Lett., 32, 425 (1995)

[15] C.A.Hurst and H.S.Green, J.Chem.Phys. 33, 1059 (1960)

[16] R.J.Baxter, "Exactly solved models in statistical mechanics", Academic Press, 1982

[17] K.Ziegler, Nucl.Phys. B 285, 606 (1987) 